\title{
6 monthly scheduled follow up of hypertension was equivalent to 3 monthly scheduled follow up
}

Birtwhistle RV, Godwin MS, Delva MD, et al. Randomised equivalence trial comparing three month and six month follow up of patients with hypertension by family practitioners. BMJ 2004;328:204-9.

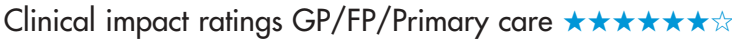

In patients with hypertension, are 3 monthly and 6 monthly scheduled follow ups by family practitioners equivalent in blood pressure (BP) control, treatment compliance, and patient satisfaction?

\section{METHODS}

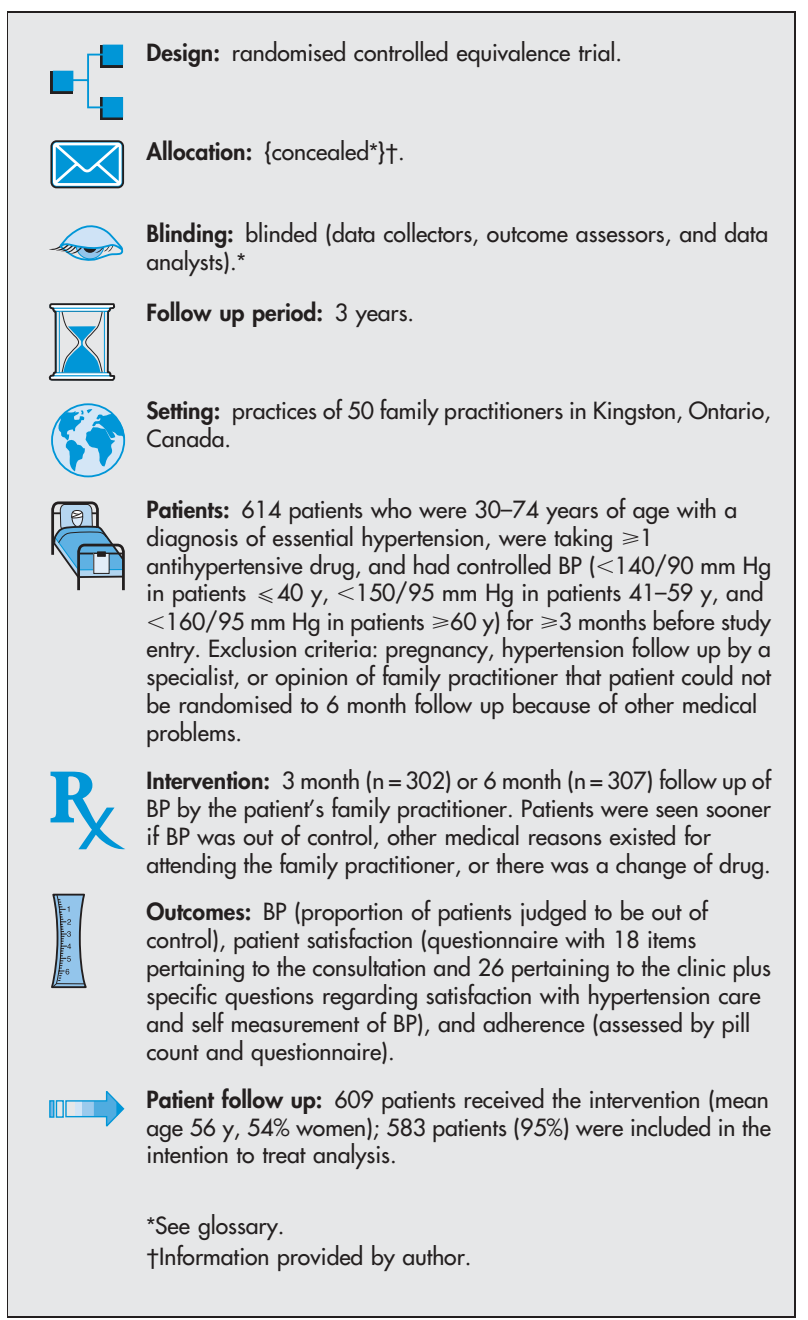

\section{MAIN RESULTS}

Patients in the 6 month follow up group had fewer visits to the family practitioner than patients in the 3 month follow up group (mean visits per patient $16.2 \vee 18.8, \mathrm{p}<0.001$ ). At baseline, 12,24 , and 36 months, mean BP measured by practitioners was equivalent between groups. The proportion of patients in whom practitioners judged BP to be out of control was similar between groups (table). The groups were equivalent for patient satisfaction with their care and wer........................................... For correspondence: Dr R Birtwhistle, Queen's University, Kingston, Ontario, Canada. birtwhis@post.queensu.ca

Sources of funding: Canadian Institute for Health Research and McKnight Fund of Queen's University.
6 month $v 3$ month follow up for hypertension judged to be out of control by family practitioners*

\begin{tabular}{llll}
\hline Follow up period & $\begin{array}{l}\text { 6 month } \\
\text { follow up }\end{array}$ & $\begin{array}{l}3 \text { month } \\
\text { follow up }\end{array}$ & Difference (95\% CI) \\
\hline 12 months & $17 \%$ & $21 \%$ & $4 \%(-2.8$ to 10$)$ \\
24 months & $23 \%$ & $20 \%$ & $3 \%(-3.3$ to 10$)$ \\
36 months & $16 \%$ & $18 \%$ & $2 \%(-8.8$ to 4$)$ \\
\hline
\end{tabular}

${ }^{*} \mathrm{Cl}$ defined in glossary. Difference and $\mathrm{Cl}$ calculated from data in article; all differences are not significant.

specifically for their hypertension care. The groups were also equivalent for adherence to treatment.

\section{CONCLUSION}

In patients with hypertension, 6 monthly scheduled follow up by family practitioners was equivalent to 3 monthly scheduled follow up for blood pressure control, treatment compliance, and patient satisfaction.

\section{Commentary}

$T$ he treatment of elevated BP is arguably the most effective preventive intervention a generalist will undertake, with the largest effect on public health. When developers of clinical guidelines formulate recommendations about management of patients with chronic illnesses or about preventive care, the duration between follow up visits is largely a matter of opinion. Investigators rarely test the effectiveness of different frequencies of follow up.

The study by Birtwhistle et al, from a mixture of rural and urban general practices in Canada, tackles this question in a randomised controlled trial, which was powered to establish equivalence in BP control and used a validated measure of patient satisfaction between patients allocated to 3 or 6 months of follow up. The study concluded that BP control and patient satisfaction were similar between the 2 groups. The difference in all consultations was only 2.5 visits over 3 years between groups.

Are the results valid for the patients in the study and can they be extrapolated to our patients? The internal validity of the study is only undermined by the relatively small difference in the number of consultations by patients in the 2 groups. Thus, interpreting equivalence in outcomes for patients with 3 and 6 month BP follow up is questionable, as is the decision to focus on BP control as the primary outcome, rather than the broader goal of management of hypertensive patients: reduction of global cardiovascular risk. Generalising the results to other patients is also problematic. Less than half the practices approached by the investigators were recruited and less than two thirds of eligible patients within those practices participated. Therefore we have to be cautious about extrapolating to all patients with elevated $\mathrm{BP}$ in general practice.

Despite these caveats, the study moves us from the realm of opinion about frequency of follow up to empirical findings. The message to clinicians is that a planned follow up every 6 months seems just as good (or bad) for blood pressure control as every 3 months, and that other considerations should be taken into account in determining when a patient should come back for review.

Gene Feder, MD, FRCGP Barts and the London, Queen Mary's School of Medicine and Dentistry London, UK 